\title{
Evaluation of different planning methods of 3DCRT, IMRT, and RapidArc for localized prostate cancer patients: planning and dosimetric study
}

\author{
Ahmed Shawky Shawata ${ }^{1}$, Mohamed Farouk Akl ${ }^{2}$, Khaled M. Elshahat ${ }^{3}$, Naer A. Baker ${ }^{4}$ and Moustafa T. Ahmed ${ }^{4^{*}}$ (D)
}

\begin{abstract}
Background: Different planning methods of IMRT planning techniques (IMRT (DMLC) and RapidArc) vs. stander techniques (three-dimensional radiation therapy (3DCRT)) will be evaluated for prostate cancer patients' planning and verification. Three groups of localized prostate cancer patients are planned and evaluated regarding DVHs and practical radiation dosimetry, and ten 3DCRT plans are assessed statistically for each patient.

Results: Plan (7) with parameters of five equally weighted fields with angles of $0^{\circ}, 45^{\circ}, 90^{\circ}, 270^{\circ}$, and $315^{\circ}$ and energy of $15 \mathrm{MV}$ is the most suitable plan both for PTV coverage and for OAR sparing with a fewer number of fields and fewer number of gantry angles. IMRT complexity involves the requirement of long treatment times and additional effort for planning, safety checks, and quality control before the patient start the treatment and proceed.

Conclusions: The selected plan also is more safe on patients up to 7400 cGy than other plans and is easier to be applied compared to IMRT and RapidArc plans, depending on patient geometry. IMRT radiation doses are more effective and can safely be delivered to PTV with little side effects compared with 3D conformal and conventional techniques. RapidArc has the advantage of re-optimizing and small arcs of variable parameters in dose delivery, taking into account the maximum speed of gantry and MLCs.
\end{abstract}

Keywords: 3DCRT, IMRT, RapidArc, DVH, Statistical analysis, Qualitative analysis

\section{Background}

Radiation therapy (radical treatment) is widely used for localized prostate curative treatment providing a reduction in late rectal toxicity compared with other conventional treatment techniques [1]. Treatment is carefully planned by using a 3D computed tomography $(\mathrm{CT})$ registered with magnetic resonance (MRI) images of the patient with computerized calculations of doses using treatment planning system (TPS) determining the various intensity patterns and tumor conformal dose [2]. Intensity-modulated radiation therapy (IMRT) is the most suitable treatment modality for the treatment of the lung, prostate, spine,

\footnotetext{
* Correspondence: moustf@mans.edu.eg

${ }^{4}$ Physics Department, Faculty of Science, Mansoura University, Mansoura, Egypt

Full list of author information is available at the end of the article
}

kidney, liver, pancreas, head, and neck cancers. IMRT comes as a solution if it is not available or appropriate for some brain cases (cannot be treated with gamma knife). IMRT is defined as a dose delivery by treatment plans that are optimized using techniques of forward or inverse planning for treatment delivery with modulated beams, using collimators or MLC shaping by modes of either step/shoot (static multileaf collimator (SMLC)) or sliding window (dynamic multileaf collimator (DMLC)) [3]. Techniques of inverse planning included signed compensators for tissue irregularity and missing tissues. Radiation therapy can be capable of destroying all cancer cells, shrinking sizes, or eliminating malignant tumor thus stopping or slowing tumor growth. Radiation is delivered by RapidArcis with gantry rotation as arc continuous on mode; various parameters will be varied (MLC shapes (apertures), dose rate output, gantry 
speed of rotation, and collimator orientation). RapidArc delivers dose distributions similar to other IMRT techniques or higher $[4,5]$. The portal image was obtained from setup fields or treatment beams using a robotic portal imager as a part of radiotherapy treatment machine (linear accelerators (LINACs)) showing that the irradiated area needs to be observed, as it is used for treatment verification, regardless of having lower image quality taken with megavolt irradiation. The portal images' main use is for the verification of patient set-up, where the patient electronic portal imaging device (EPID) images are matched with anterior and lateral DRR reference images for position verification of patient. Matched images were verified in reference to the patient's bony landmarks giving the organ position; it is not surely static in the same position relative to the bone. Different ways of verification using information from portal dose are comparing the acquired image and the measured dose image from the portal or by the back projection of information from transmitted dose to calculate patient dose compared to treatment plan dose distribution [6]. These methods may reduce errors in MLC positioning and movement and the LINAC dosimetry and mechanical performance providing a high confidence level for plan accuracy before patient treatment [7].

\section{Objective of the study}

This study aims to evaluate different planning methods of IMRT planning techniques (forward planning with three-dimensional radiation therapy (3DCRT), inverse planning with IMRT (DMLC), and RapidArc) for cancer patients. IMRT is very advanced; it needs more quality and accuracy in evaluation before treatment due to many small fields included in plans and different dose areas in the same plan as we have to be sure that the planning encompasses the volumes as prescribed or not. So we did the following:

1. Patient selection and treatment sites (prostate)

2. We studied some images indicative of the tumor

3. Pre-treatment planning

4. Final treatment planning (3DCRT, IMRT, and Rapid Arc) is performed

5. We evaluated different methods of treatment planning:

- To compare different DVHs for all plans (Eclipse and Prowess planning systems)

- To use tools of evaluation like biological optimization and physical optimization

- To conclude and recommend the most suitable treatment methods

\section{Materials and methods}

\section{Patients}

We choose three groups of prostate patients (20 patients with volumes ranging from 23 to $76 \mathrm{cc}$ ). We included a group of patients with intermediate-risk prostate cancer which are patients with clinical stages T2b to T2c, Gleason score 7, or PSA values 10 to $20 \mathrm{ng} / \mathrm{ml}$. CT simulation is performed for all patients with $3-\mathrm{mm}$ slice thickness under the same protocol (positioned in the supine position). Immobilization was done by the knee and ankle support to ensure fixation and reproducibility; the patient arms are on the chests. We scanned the patient by $\mathrm{CT}$ from the abdomen till the mid-thigh to allow contouring all the organs at risk. All patients were immobilized with bladder comfortably full and empty rectum; it is a must in every treatment secession as it is done first before CT scan and setup. The clinical target volume (CTV) included the whole prostate gland and the proximal $1 \mathrm{~cm}$ of the seminal vesicles. We created PTV by extending the CTV by $1 \mathrm{~cm}$ in all directions except posteriorly (only $6 \mathrm{~mm}$ ) to decrease dose to the rectum, and also, organs at risk (OARs) (bladder, rectum, and both left and right femoral heads) are outlined. The prescribed dose is $(74 \mathrm{~Gy} / 7.5$ weeks/37 fractions) with a secession dose limit of $200 \mathrm{cGy} / \mathrm{Fr}$.

\section{Beam arrangement}

Varian (TrueBeam) linear accelerator with the Eclipse planning system is used. All beam arrangements were visualized using the beam's eye view (BEV) display. MLC beam shaping was used to create beam apertures. Asymmetric collimation and other parameters were used when necessary with 3DCRT, IMRT, and RapidArc plans.

We were able to deliver dose to the prostate up to 7400 cGy with 3DCRT plans. The X-ray beam energy used was $6 \mathrm{MV}$ or $15 \mathrm{MV}$ when needed regarding the effective path with tissue density correction. Ten 3DCRT plans used as forward plans for each patient with coplanar beam arrangement techniques were designed. Plan (1) was arranged by angles of $0^{\circ}, 120^{\circ}$, and $240^{\circ}$ : anterior direct with an energy of $6 \mathrm{MV}$ and two lateral (Rt and Lt) oblique wedged (W15) beams with an energy of $15 \mathrm{MV}$. Plan (2) was arrange by angles of $0^{\circ}, 90^{\circ}$, and $270^{\circ}$, anterior direct and two lateral (Rt and Lt) wedged (W60) beams, all beams with an energy of 15MV. Plan (3) was arranged by angles of $0^{\circ}, 90^{\circ}, 180^{\circ}$, and $270^{\circ}$ : anterior direct with an energy of $6 \mathrm{MV}$, posterior direct with an energy of $15 \mathrm{MV}$, and two lateral (Rt and Lt) oblique beams with an energy of $15 \mathrm{MV}$, all open without wedges. Plan (4) was arranged by angles of $45^{\circ}$, $315^{\circ}, 135^{\circ}$, and $225^{\circ}$ : two anterior oblique wedged beams (W30 L and W30R are directed with the thin end down and the thick end up) with an energy of $6 \mathrm{MV}$ and two 


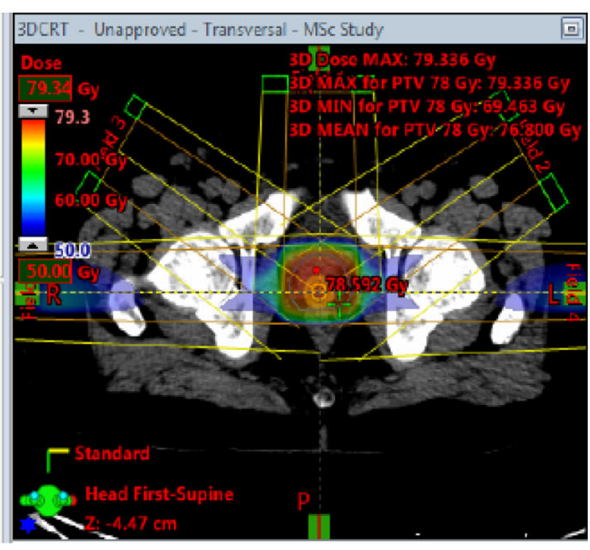

A

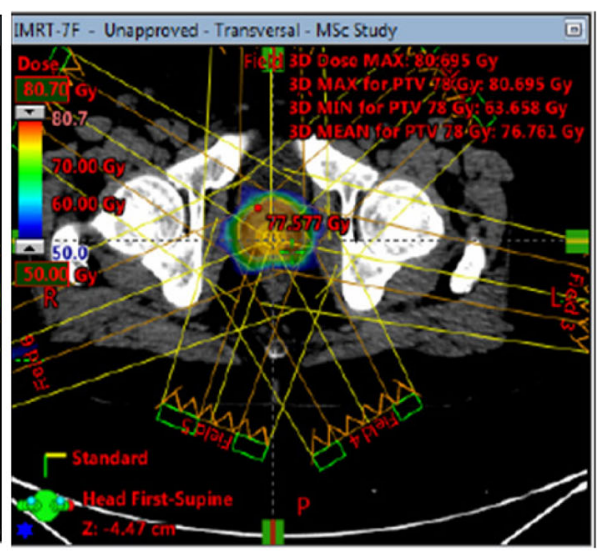

B

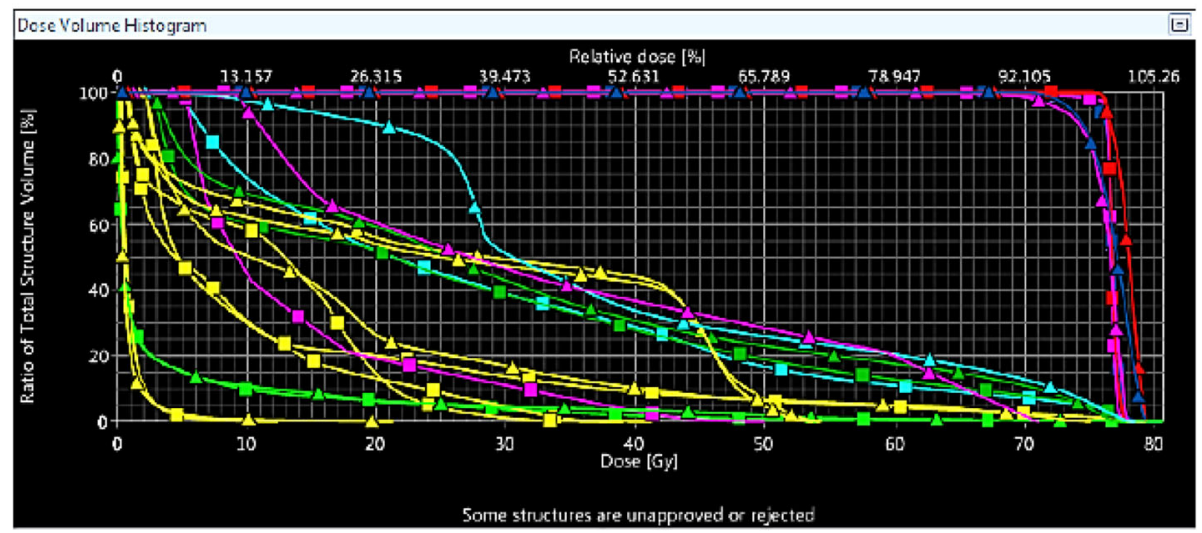

C

Fig. 1 Both a 3DCRT plan and b IMRT plan was evaluated according to $\mathbf{c}$ dose-volume histogram (DVH) comparison displaying all organs (PTV, rectum, bladder, Rt femoral head, and Lt femoral head)

posteriors (Rt and Lt) oblique beams with an energy of $15 \mathrm{MV}$. Plan (5) was arranged by angles of $0^{\circ}, 45^{\circ}, 315^{\circ}$, $135^{\circ}, 180^{\circ}$, and $225^{\circ}$ : all fields are open without wedges and with an energy of $15 \mathrm{MV}$. Plan (6) was arranged by angles of $0^{\circ}, 60^{\circ}, 120^{\circ}, 240^{\circ}$, and $300^{\circ}$ : all fields are open without wedges and with an energy of $15 \mathrm{MV}$. Plan (7) is a five-field technique and was arranged by angles of $0^{\circ}$, $45^{\circ}, 90^{\circ}, 270^{\circ}$, and $315^{\circ}$ : all fields are open without wedges and with an energy of $15 \mathrm{MV}$. Plan (8) was arranged by angles of $0^{\circ}, 60^{\circ}, 90^{\circ}, 120^{\circ}, 220^{\circ}, 270^{\circ}$, and $300^{\circ}$ : one anterior direct beam, two anterior oblique beams with an energy of $6 \mathrm{MV}$, two direct lateral (Rt and Lt) wedged (W60L and W60R) beams with an energy of $6 \mathrm{MV}$, and two posterior (Rt and Lt) oblique beams with an energy of $15 \mathrm{MV}$. Plan (9) is an eight fields technique (with MLC) were arranged by angles of $0^{\circ}, 45^{\circ}, 90^{\circ}, 135^{\circ}$, $180^{\circ}, 225^{\circ}, 270^{\circ}$, and $315^{\circ}$ : one anterior direct beam, two anterior oblique beams, two direct lateral (Rt and Lt) wedged (W30 L and W30R are directed with the thin end down and the thick end up) beams, one posterior direct beam, and two posterior (Rt and Lt) oblique, all beams are with an energy of $15 \mathrm{MV}$. Plan (10) is a plan (9) but without MLC. Plan normalization is set to $100 \%$ at beam isocenter, and the PTV is covered by more than 95\% isodose; the PTV maximum dose is ranging around $101 \%$. We delivered the dose to the prostate up to 7400 cGy prescription dose by IMRT and RapidArc plans; all beams with an energy of $6 \mathrm{MV}$. The six-field technique was arranged by angles of $0^{\circ}, 45^{\circ}, 90^{\circ}, 180^{\circ}, 270^{\circ}$, and $315^{\circ}$ with 8 segments per beam (total number of beamlets is 48 openings) and collimator angle of $0^{\circ}$, maximum $\mathrm{MU} / \mathrm{Fr}$ of $90 \mathrm{MU}$, and minimum segment area of $1 \mathrm{~cm}^{2}$. The seven-field technique were arranged by angles of $0^{\circ}$, $50^{\circ}, 90^{\circ}, 130^{\circ}, 230^{\circ}, 270^{\circ}$, and $310^{\circ}$ with 6 segments per beam (total number of beamlets is 42 openings) and collimator angle of $0^{\circ}$, maximum MU/Fr of $60 \mathrm{MU}$, and minimum segment area of $1 \mathrm{~cm}^{2}$. Another seven-field technique was arranged by angles of $0^{\circ}, 51^{\circ}, 103^{\circ}, 155^{\circ}$, $206^{\circ}, 257^{\circ}$, and $308^{\circ}$ with 5 segments per beam (total number of beamlets is 35 openings) and collimator angle of $90^{\circ}$, maximum $\mathrm{MU} / \mathrm{Fr}$ of $60 \mathrm{MU}$, and minimum segment area of $1 \mathrm{~cm}^{2}$. While planning, we can increase 


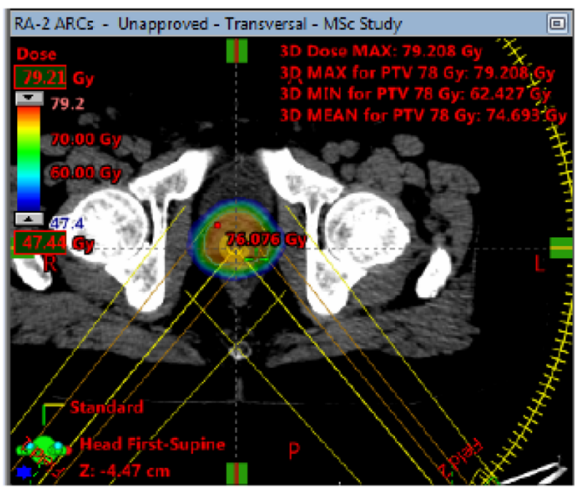

A

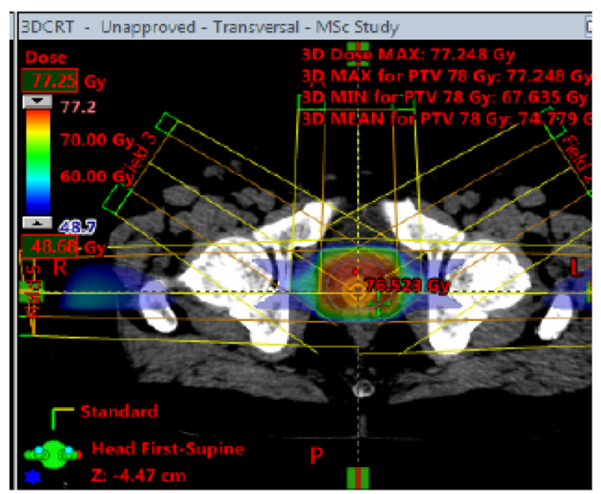

B

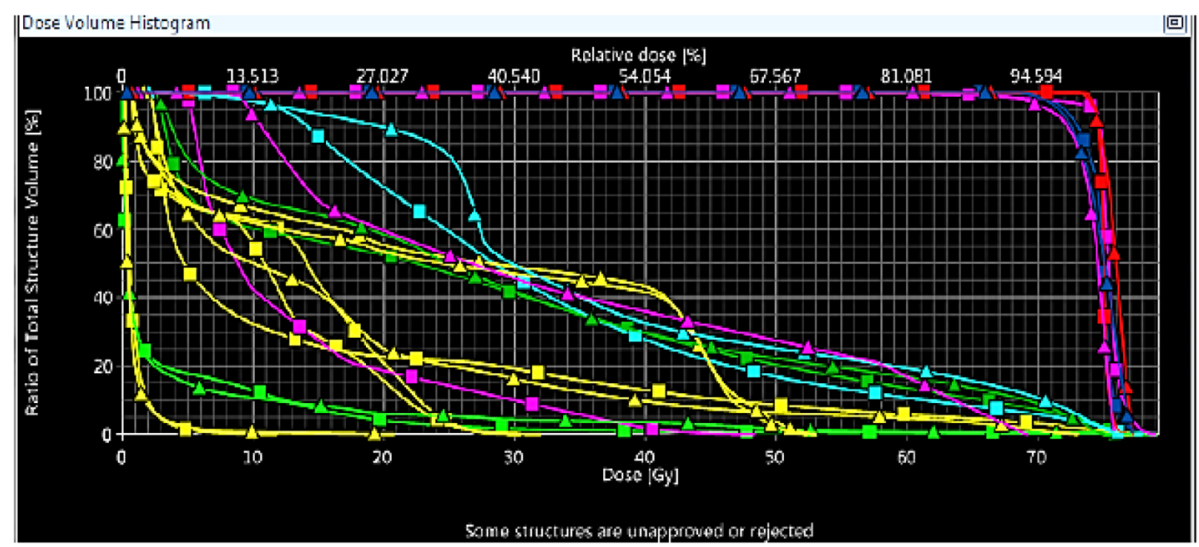

C

Fig. 2 Both a RapidArc plan and b 3DCRT plan were evaluated according to $\mathbf{c}$ dose-volume histogram (DVH) comparison displaying all organs (PTV, rectum, bladder, Rt femoral head, and Lt femoral head)

the penalty weight for both femoral heads until hot spots can reach the acceptable dose limit as hot spots appear near the PTV surface and both femora dose limits may exceed the constraint, taking a long time to achieve optimization. The RapidArc plan was designed with two $\operatorname{arcs}\left(179.0^{\circ} \mathrm{CCW}\right.$ to $181.0^{\circ}$ and $181.0^{\circ} \mathrm{CW}$ to $\left.179.0^{\circ}\right)$ with an energy of $10 \mathrm{MV}$.

\section{Results}

The ten plans were investigated regarding acceptance limits with DVH evaluation, and all results are statistically analyzed before a final decision for an optimal plan that can be accepted with the highest prescribed dose. Figure 1 shows the DVH comparison and plan evaluation for the 3DCRT plan (7) and IMRT plan displaying all organs, showing the plan acceptance, and satisfying all dose limits, saving all OARs; the PTV volume of 95\% is covered by $95.9 \%$ of dose-saving all of the rectum, bladder, Rt femoral head, and Lt femoral head. The femoral head dose limits used for acceptance are dose maximum $\left(D_{\max }\right)$ $<30 \mathrm{~Gy}$, mean dose $<2.5 \mathrm{~Gy}$, and V $40<40 \%$. The bladder dose limits are $D_{\max }<65 \mathrm{~Gy}, \mathrm{~V} 65 \leq 50 \%$, and $\mathrm{V} 70 \leq 35 \%$.
Rectum dose limits are V50 < 50\%, V60 < 35\%, V65 < 25\%, and $\mathrm{V} 70<20 \%$.

The RapidArc plan and 3DCRT plan evaluations displaying all organs (PTV, rectum, bladder, Rt femoral head, and Lt femoral head) are shown in Fig. 2. Also, the RapidArc and IMRT plan evaluations with DVH comparison displaying all organs (PTV $74 \mathrm{~Gy}$, rectum, bladder, Rt femoral head, and Lt femoral head) are shown in Fig. 3.

The IMRT and RapidArc plans achieved the following constraints regarding prostate PTV: penalize all points outside $7644.00 \mathrm{cGy}$ and $8034.00 \mathrm{cGy}$ with the weight of 100.00 , penalize if $<99.00 \%$ of the volume is below 7644.00 cGy with the weight of 100.00 , and penalize if $>1.00 \%$ of the volume is above 8190.00 cGy with the weight of 100.00 . But for critical organs like bladder, penalize if $>15.00 \%$ of volume is above 8002.80 cGy with a weight of 20.00 , penalize if $>25.00 \%$ of volume is above 7503.60 cGy with a weight of 20.00 , penalize if $>50.00 \%$ of volume is above $6497.40 \mathrm{cGy}$ with a weight of 20.00 , penalize if $>20.00 \%$ of volume is above 5499.00 cGy with a weight of 20.00 , and penalize if $>1.00 \%$ 


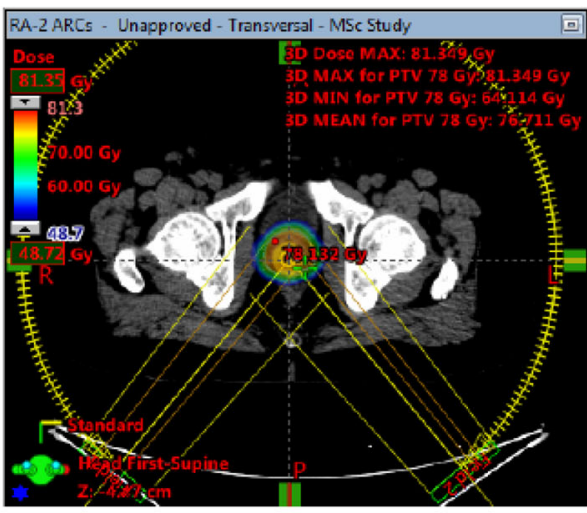

A

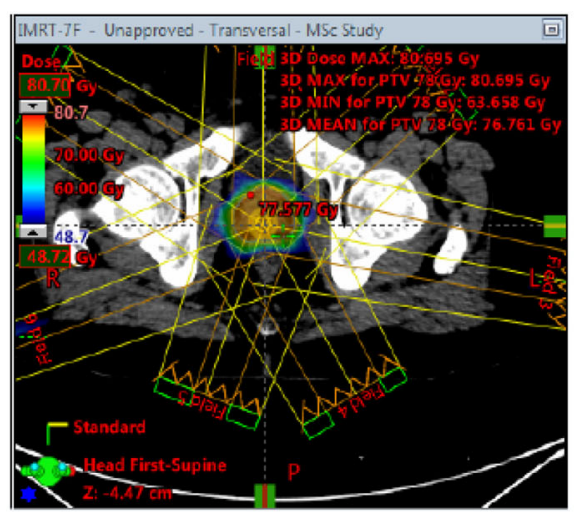

B

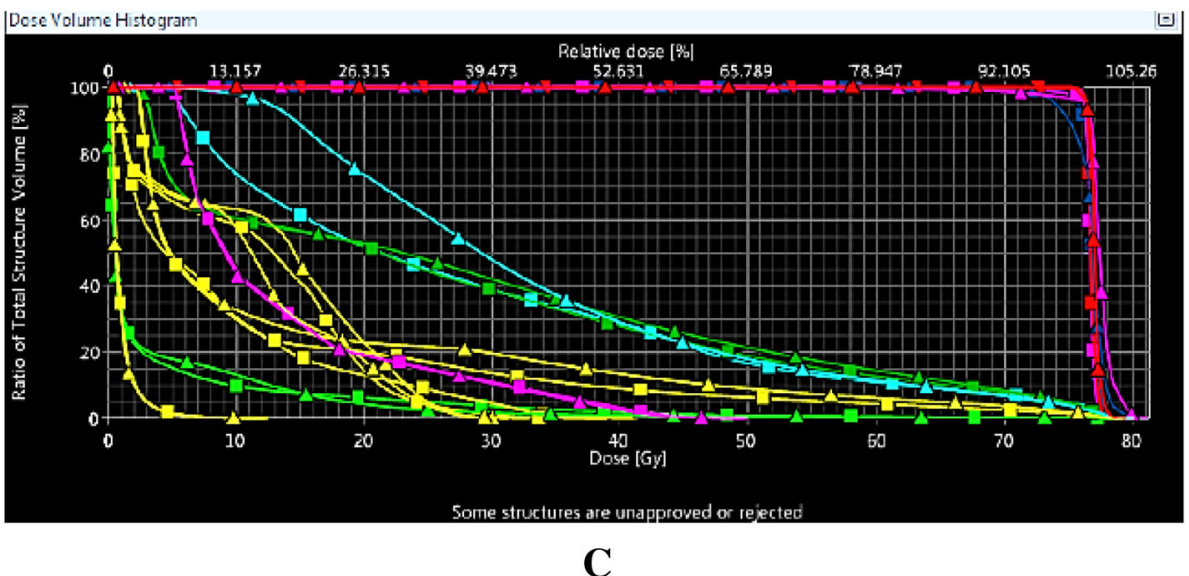

Fig. 3 Both a RapidArc plan and b IMRT plan was evaluated according to c dose-volume histogram (DVH) comparison displaying all organs (PTV, rectum, bladder, Rt femoral head, and Lt femoral head)

of volume is above 7503.60 cGy with a weight of 78.00 . And for rectum, penalize if $>15.00 \%$ of the volume is above 7503.60 cGy with the weight of 40.00 , penalize if $>25.00 \%$ of the volume is above $6497.40 \mathrm{cGy}$ with the weight of 40.00 , and penalize if $>50.00 \%$ of the volume is above 5499.00 cGy with the weight of 40.00 , as rectum constraints are used if the rectum is delineated from the anus to the sigmoid and also if the rectum intersects with PTV with too high volume $(<20 \%$ of the rectum volume). For $\mathrm{Rt}$ femur, penalize all points $>4500.60$ cGy with the weight of 50.00 and penalize all points $>3502.20$ cGy with the weight of 20.00. And for Lt femur, penalize all points $>4500.60 \mathrm{cGy}$ with the weight of 50.00 and penalize all points $>3502.20 \mathrm{cGy}$ with the weight of 20.00 .

\section{Discussions}

Statistical analysis and qualitative analysis for PTV and OAR with delivered dose by the ten 3D plans are discussed. DVH analysis for the ten plans regarding PTV (min, max, mean, V90, and V95 doses) is assessed in Fig. 4 as organs at risk are not exceeding their tolerances [8]. From the statistical analysis, the best dose coverage regarding PTV $\min$ is for plan (6) that shows the mean dose of 6648 cGy, but regarding PTV mean dose, we found that the best dose coverage is for plan (9) with the mean of 7437 cGy and the plan (5) mean dose is $99 \%$ within the interval between $98 \%$ and $99.7 \%$, showing the accepted dose coverage for PTV with fewer number of fields helping in easier and faster dose delivery. Regarding the PTV dose of $95 \%$, the best result is for plan (7) showing the mean of 7178 cGy within the interval of 7098 cGy-7256 cGy with a $p$ value of 0.4 , but also, plan (5) shows the mean dose of $7055 \mathrm{cGy}$ ( $95 \%$ of dose) within the interval between $94.2 \%$ and $96.5 \%$ of the total dose.

Plan (7) is the best for PTV dose coverage with a $95 \%$ isodose line of $7178 \pm 117 \mathrm{cGy}, 95 \%$ CI of $7098-7256$, and $P=0.4$. Plan (10) is the lowest PTV dose coverage with a $95 \%$ isodose line of $7031 \pm 257$ cGy, $95 \%$ CI of 6858-7203, and $P=0.4$. Plan (9) is the best for PTV with a mean dose of $7437 \pm 125$ cGy, 95\% CI of 73537520 , and $P=0.01$. Plan (9) is the lowest PTV with a mean dose of $7232 \pm 105$ cGy, 95\% CI of 7152-7292, and $P=0.01$. Also, plan (5) is covered by $99 \%$ mean dose 


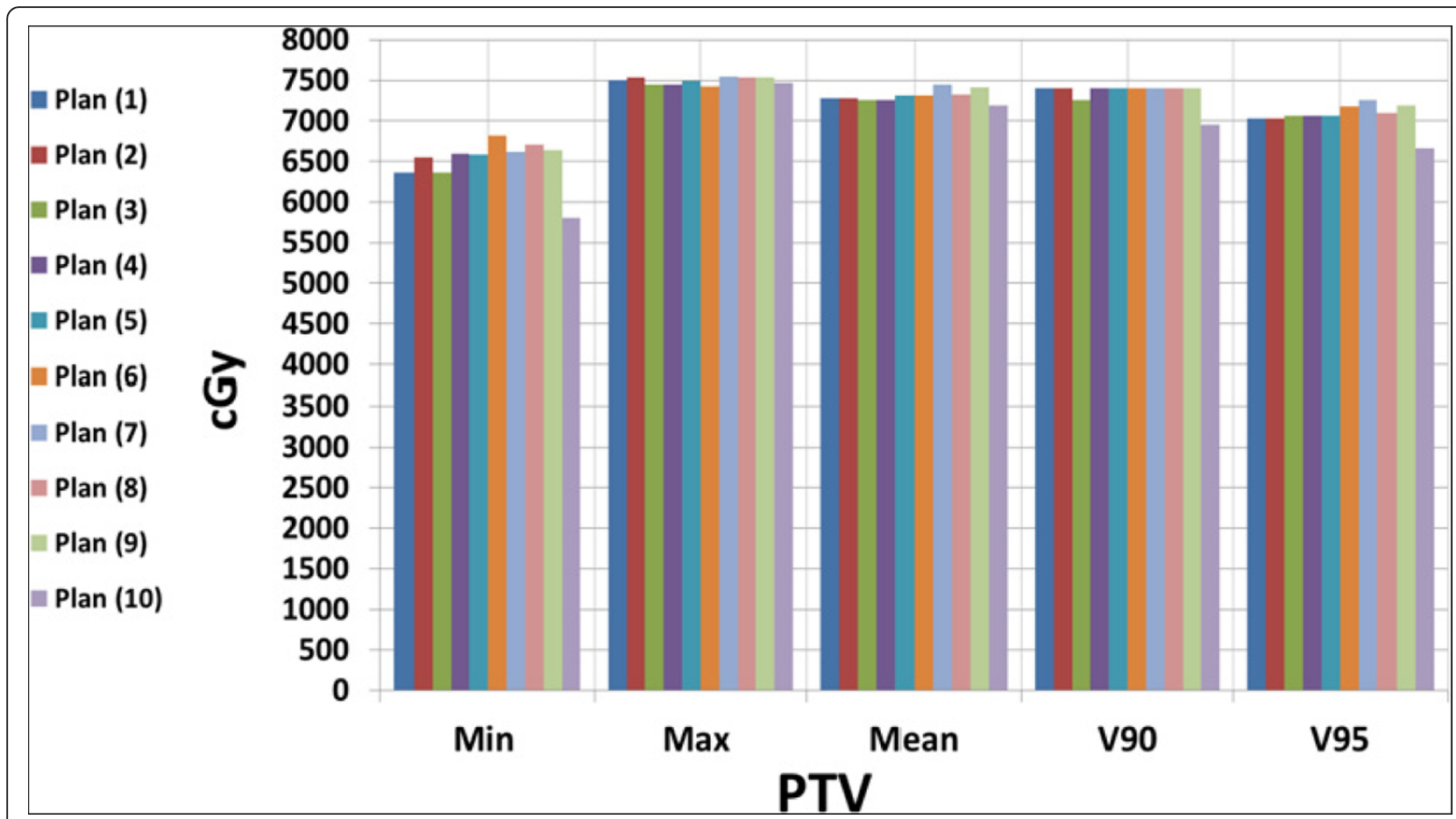

Fig. 4 DVH analysis for the ten plans regarding PTV (min, max, mean, V90, and V95 doses)

of $7328 \pm 78$ cGy, $95 \% \mathrm{CI}$ of $7275-7380$, and $P=0.01$; this plan is accepted for treatment, saving much time with a lower number of fields. Regarding PTV minimum dose, plan (6) has the highest PTV dose coverage of $6648 \pm 248$ cGy, 95\% CI of 6481-6815, and $P=0.01$. Plan (10) has the lowest PTV minimum dose coverage of $5873 \pm 489$ cGy, large scale $95 \% \mathrm{CI}$ of $5545-6201$, and $P=0.01$. The DVH analysis for the ten plans regarding the bladder (max, mean, V70, V65, V60, and V50 doses) is assessed in Fig. 5a. Plan (7) showed the lowest dose to the bladder with a maximum dose of $6298 \pm 2301$ cGy, satisfying the condition of maximum dose <65 Gy, and plan (5) also satisfied the same condition for some plans depending on the prostate volume with $P=0.32$. All the ten plans satisfied the critical dose limits of (V70 $\leq 35 \%$ and V65 $\leq 50 \%)$ as all plans are accepted for treatment. Plan (7) showed acceptable results for both the right and left femoral heads; the Lt femur maximum dose is $3036 \pm$ 731 cGy, with $95 \% \mathrm{CI}$ of $2545-3527$ and $P=0.00$, the Lt femur mean dose is $1030 \pm 698$ cGy, with $95 \%$ CI of 560 1499 and $P=0.00$, the Rt femur maximum dose was 2814 \pm 700 cGy, with $95 \%$ CI of $2343-3283$ and $P=0.00$, and the Rt femur mean dose is $953 \pm 456$ cGy, with $95 \% \mathrm{CI}$ of 647-1259 and $P=0.00$.

Plan (5) showed acceptable doses for the right and left femoral heads with $P=0.00$; the Lt femur maximum dose is $3320 \pm 180$ cGy with $95 \%$ CI of $2108-4530$, the Lt femur mean dose is $566 \pm 623$ cGy with $95 \%$ CI of
147-985, and the Rt femur mean dose is $643 \pm 377$ cGy with $95 \%$ CI of 389-896. But plan (7) showed better results than plan (5). All plans satisfied the femoral head dose limit of V40 < 40 Gy. All plans satisfied the tolerance table dose constraints for rectum dose limit of V $75<15 \%$, and the rectum dose was accepted for treatment as the rectum was empty (volume range is $29.8-100.6 \mathrm{~cm}^{2}$ ), saving the rectum and reducing rectal toxicity as seen in Fig. 5b. IMRT radiation doses are more effective and can safely be delivered to planning target volumes (PTV) with little side effects compared with 3D conformal and conventional techniques. IMRT complexity involves the requirement of long treatment times and additional effort for planning, safety checks, and quality control before the patient starts the treatment and proceed. Increasing the total radiation dose to the tumor with more sparing for normal tissues and also with more conformity for PTV can be achieved using IMRT better than using conventional or conformal radiotherapy [9]. The overall treatment aims to achieve the goal of full tumor control during every fraction with cancer patients $[10,11]$. With the presence of metastatic cancers or continues growth of tumor with other added symptoms, the patient may die due to any failure in tumor full control. All plans were verified using practical radiation dosimetry systems like EPID (for 3DCRT, IMRT) with an acceptance range of 95-99.1\% and Delta ${ }^{4}$ (for RapidArc) with an acceptance range of $98.5-100 \%)[7,10,12]$. 
A

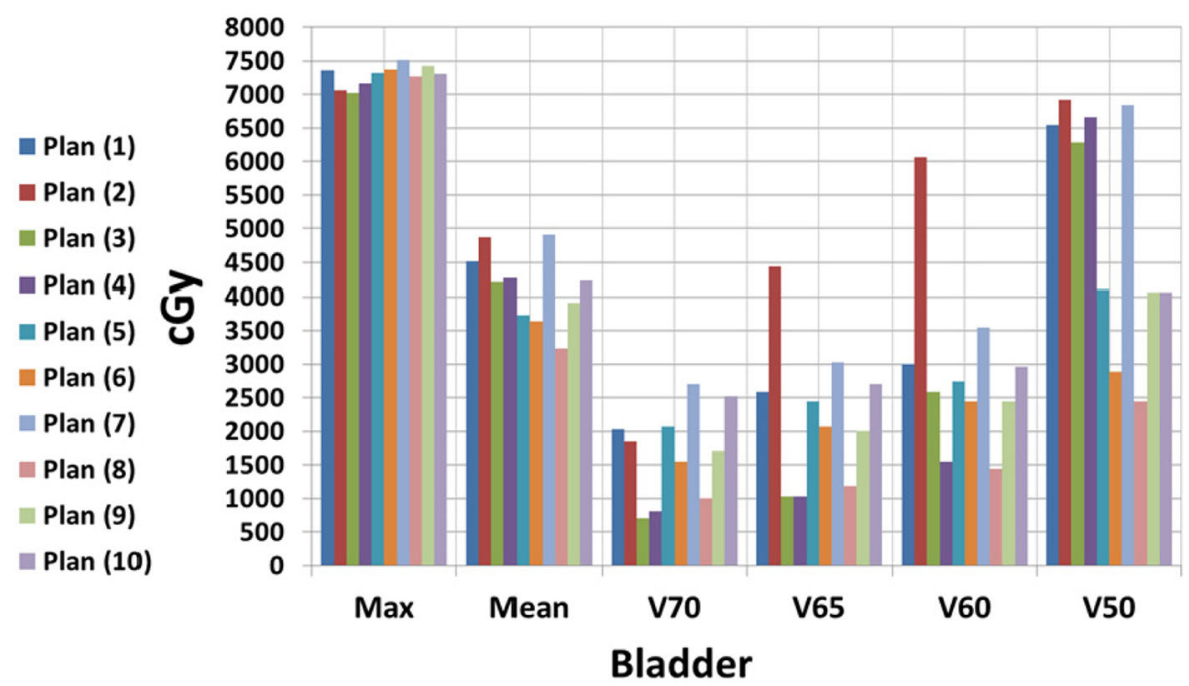

B

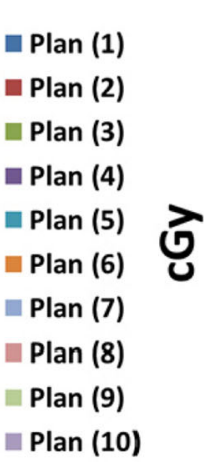

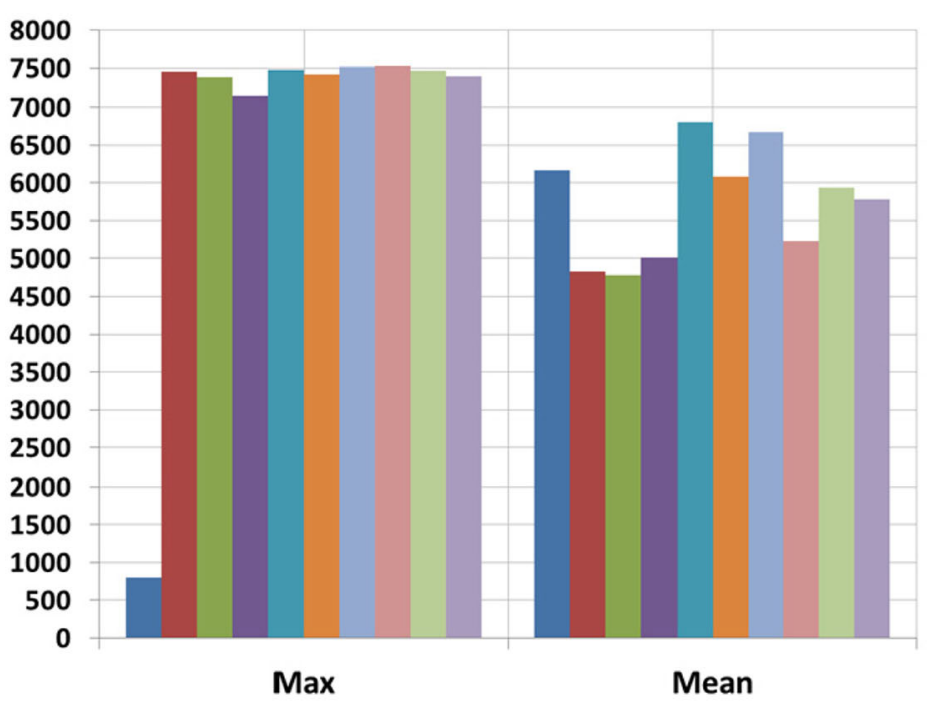

Rectum

Fig. $5 \mathrm{DVH}$ analysis for the ten plans regarding a the bladder (max, mean, V70, V65, V60, and V50 doses) and $\mathbf{b}$ rectum (max, mean)

\section{Conclusion}

IMRT radiation doses are more effective and can safely be delivered to planning target volumes (PTV) with little side effects compared with 3D conformal and conventional radiotherapy techniques as the ratio of normal tissue dose to tumor dose is reduced. IMRT complexity involves the requirement of long treatment times and additional effort for planning, safety checks, and quality control before the patient starts the treatment and proceed. Using IMTR helps us to achieve the desire to increase tumor doses and decrease dose to normal organs. The limited use of both IMRT and RapidArc plans is proportional to the high cost of required equipment, operations, and experienced team costs. For prostate treatment, plan (7) with parameters of five equally weighted fields with angles of $0^{\circ}, 45^{\circ}, 90^{\circ}, 270^{\circ}$, and $315^{\circ}$ and an energy of $15 \mathrm{MV}$ is the most suitable plan both for PTV coverage and for OAR sparing and also saving more time as all beams are open without using wedges as fewer number of fields and fewer number of movements (gantry angles) save a lot of time during treatment, saving patients' time and saving the treatment center time as well as giving the chance for other patients to be treated. The selected plan also is more 
safe on patients up to 74 Gy compared to IMRT and RapidArc plans, reducing normal tissue-irradiated volume and reducing toxicity as one of IMRT disadvantages is the presence of secondary malignancies due to the large irradiated volume with low doses during treatment. RapidArc has the advantage of re-optimizing and small arcs of variable parameters in dose delivery, taking into account the maximum speed of gantry and MLCs.

\section{Abbreviations}

3DCRT: Three-dimensional radiation therapy; BEV: Beam's eye view; CCW: Counterclockwise; CT: Computed tomography; CTV: Clinical target volume; CW: Clockwise; $D_{\max }$ : Dose maximum; DMLC: Dynamic multileaf collimator; DRR: Digitally reconstructed radiograph; DVH: Dose-volume histogram; EPID: Electronic portal imaging device; Fr: Fraction; Gy: Gray; IMRT: Intensity-modulated radiation therapy; LINAC: Linear accelerators; Lt: Left; MU: Monitor unit; OAR: Organ at risk; PSA: Prostate-specific antigen; PTV: Planning target volume; Rt: Right; SMLC: Static multileaf collimator; TPS: Treatment planning system

\section{Acknowledgements}

All of the authors thank the anonymous reviewers for their helpful comments on the original manuscript. Also, we thank Dr Ahmed Ramadan Eldesoky 'MD' (Clinical Oncology and Nuclear Medicine Department, Faculty of Medicine, Mansoura University) for his help.

\section{Authors' contributions}

KME and ASS carried out the planning processes and studies, participated in the sequence alignment, and drafted the manuscript. MFA selected the patients for the study and carried out the planning target volumes and organ at risk delineation. MFA and ASS performed the statistical analysis. NAB participated in the sequence alignment and participated in the drafting of the manuscript. MTA conceived of the study, participated and supervised in the manuscript design and coordination, and helped to draft it. All authors read and approved the final manuscript.

\section{Funding}

This study had no funding from any resource.

\section{Availability of data and materials}

The datasets used and/or analyzed during the current study are available from the corresponding author on reasonable request.

\section{Ethics approval and consent to participate}

It is "not applicable" as we did not try the treatment on real patients, and it is only a dosimetric study on a computer.

\section{Consent for publication}

It is "not applicable." We did not ask all the patients included in this research to give written informed consent to publish the data contained within this study as we used their data only for research without application of plans on them (dosimetric study on planning system only).

\section{Competing interests}

The authors declare that they have no competing interests.

\begin{abstract}
Author details
${ }^{1}$ Radiation Oncology Center, Tanta Military Hospital, Tanta, Egypt. ${ }^{2}$ Clinical Oncology and Nuclear Medicine Department, Faculty of Medicine, Mansoura University, Mansoura, Egypt. ${ }^{3}$ Clinical Oncology Department, Faculty of Medicine, Al Azhar University, Cairo, Egypt. ${ }^{4}$ Physics Department, Faculty of Science, Mansoura University, Mansoura, Egypt.
\end{abstract}

Received: 10 June 2019 Accepted: 22 July 2019

Published online: 07 September 2019

\section{References}

1. Di Franco R, Borzillo V, Ravo V, Ametrano G, Falivene S, Cammarota F, Rossetti S, Romano FJ, D'Aniello C, Cavaliere C, lovane G, Piscitelli R, Berretta
M, Muto P, Facchini G (2017) Rectal/urinary toxicity after hypofractionated vs conventional radiotherapy in low/intermediate-risk localized prostate cancer: systematic review and meta-analysis. Oncotarget 8(10):17383-17395

2. Pinnaduwage DS, Descovich M, Lometti MW, Varad B, Roach M, Gottschalk AR (2017) An evaluation of robotic and conventional IMRT for prostate cancer: potential for dose escalation. Technol Cancer Res Treat 16(3):267-275

3. Salimi M, Abi KST, Nedaie HA, Hassani H, Gharaati H, Samei M, Shahi R, Zarei H (2017) Assessment and comparison of homogeneity and conformity indexes in step-and-shoot and compensator-based intensity modulated radiation therapy (IMRT) and three-dimensional conformal radiation therapy (3D CRT) in prostate cancer. J Med Signals Sensors 7(2):102-107

4. White P, Chan KC, Cheng KW, Chan KY, Chau MC (2013) Volumetric intensity-modulated arc therapy vs conventional intensity-modulated radiation therapy in nasopharyngeal carcinoma: a dosimetric study. J Radiat Res 54(3):532-545

5. Huang B, Fang Z, Huang Y, Lin P, Chen Z (2014) A dosimetric analysis of volumetric- modulated arc radiotherapy with jaw width restriction vs 7 fields intensity-modulated radiotherapy for definitive treatment of cervical cancer. Br J Radiol 87(1039):20140183

6. Huang M, Huang D, Zhang J, Chen Y, Xu B, Chen L (2017) Preliminary study of clinical application on IMRT three-dimensional dose verification-based EPID system. J Appl Clin Med Phys 18(4):97-105

7. Kim J, Park S-Y, Kim HJ, Kim JH, Ye S-J, Park JM (2014) The sensitivity of gamma-index method to the positioning errors of high-definition MLC in patient-specific VMAT QA for SBRT. Radiat Oncol (London, England) 9:167

8. Lu JY, Wu LL, Zhang JY, Zheng J, Cheung ML, Ma CC, Xie LX, Huang BT (2015) Improving target dose coverage and organ-at-risk sparing in intensity-modulated radiotherapy of advanced laryngeal cancer by a simple optimization technique. Br J Radiol 88(1046):20140654

9. Betzel GT, Yi BY, Niu Y, Yu CX (2012) Is RapidArc more susceptible to delivery uncertainties than dynamic IMRT? Med Phys 39(10):5882-5890

10. Cozzolino M, Oliviero C, Califano G, Clemente S, Pedicini P, Caivano R, Chiumento C, Fiorentino A, Fusco V (2014) Original paper: clinically relevant quality assurance (QA) for prostate RapidArc plans: gamma maps and DVH -based evaluation. Phys Med 30:462-472

11. Vieillevigne L, Molinier J, Brun T, Ferrand R (2015) Gamma index comparison of three VMAT QA systems and evaluation of their sensitivity to delivery errors. Phys Med 31:720-725

12. Son J, Baek T, Lee B, Shin D, Park SY, Park J, Lim YK, Lee SB, Kim J, Yoon M (2015) A comparison of the quality assurance of four dosimetric tools for intensity modulated radiation therapy. RadiolOncol 49:307-313

\section{Publisher's Note}

Springer Nature remains neutral with regard to jurisdictional claims in published maps and institutional affiliations.

\section{Submit your manuscript to a SpringerOpen ${ }^{\circ}$ journal and benefit from:}

- Convenient online submission

- Rigorous peer review

- Open access: articles freely available online

- High visibility within the field

- Retaining the copyright to your article

Submit your next manuscript at $\boldsymbol{\nabla}$ springeropen.com 